\title{
NEW APPROACH TOWARDS EFFICIENT BIOMETRIC SYSTEMS
}

\author{
Chander Kant
}

Kurukshetra University

Kurukshetra, Haryana, India

ckverma@rediffmail.com

\begin{abstract}
Fingerprints possess two main types of features that are used for automatic fingerprint identification and verification: (i) Ridge and Furrow structure that forms a special pattern in the central region of the fingerprint and (ii) Minutiae details associated with the local ridge and furrow structure. In a traditional biometric recognition system, the biometric template is usually stored on a central server during enrollment. The candidate biometric template captured by the biometric device is sent to the server where the processing and matching steps are performed. The proposed work presents an approach to the processing time during fingerprint matching process in a Biometric System. The proposed work is based upon four major classifications of fingerprint, whorl, arch, left-loop and right-loop and is more efficient as compared with the existing system.
\end{abstract}

Keywords: Biometrics, identification, verification, minutiae points, singular points.

\section{INTRODUCTION}

Fingerprint Identification is the method of useridentification by making use of the fingerprint impressions. The method usually works on the basis of minutiae points found on the fingerprint. No two persons have exactly the same arrangement of ridge patterns, and the patterns of any one individual remain unchanged throughout life. Fingerprints offer foolproof means of personal identification.

Fingerprint identification process can be carried out in two ways [1]: minutiae-based and correlation based. The commonly used approach with minimum FAR (false accept rate) and minimum FRR (false reject rate) is minutiae based technique. In this process, first minutiae points are calculated and then are mapped their relative placement on the finger.

Fingerprint Identification System determines the matched template of input fingerprint with a stored fingerprint template in Database. The Database of templates is obtained by fingerprint enrollment process. The Identification process starts by putting the user's fingerprint at sensor level. The input fingerprint then processed in various step like; acquisition, pre-processing, feature extraction and matching as shown in Figure 1. In acquisition stage the biometric system get the input template using sensor; preprocessing includes image enhancement, gray level adjustment, ridge thinning, etc.

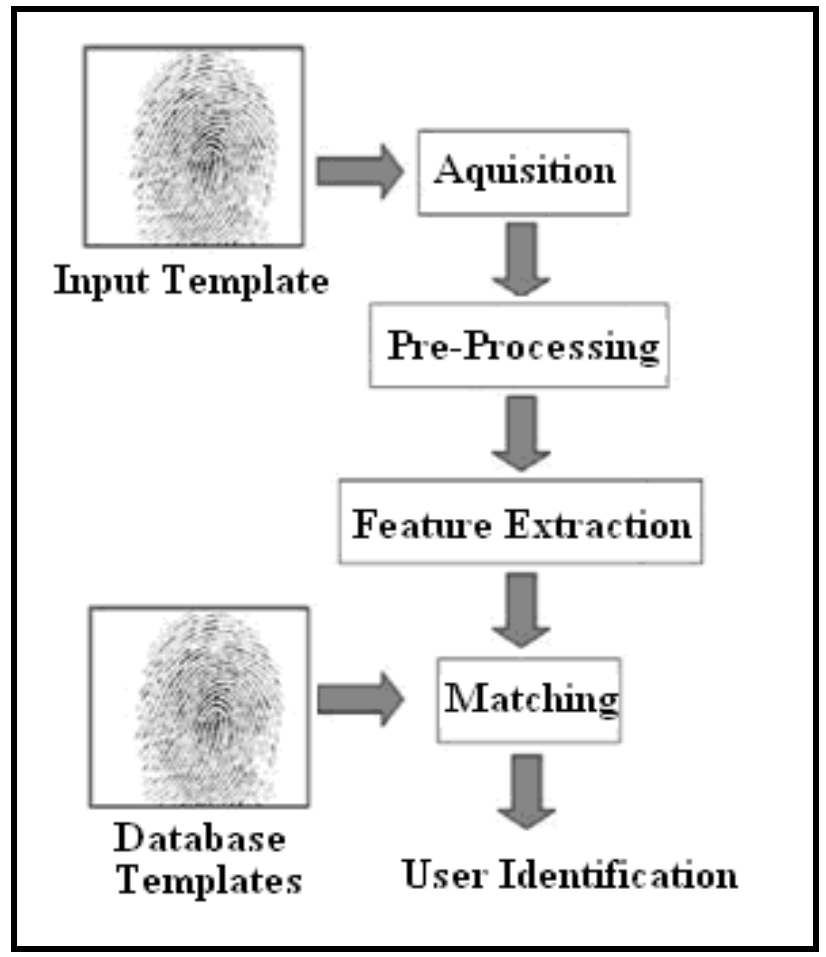

Fig. 1 - Block diagram of Fingerprint Identification system

After having the fingerprint image preprocessed, the feature extraction block extracts the minutiae points from template and then the extracted minutiae point undergoes with a matching process to match 
with the stored database Templates and finally identify the person if it is in system database. The database of biometric system plays very important role in identification process as all the templates are stored in it. The system performance also depends on the architecture of system database that is the way templates are stored there in. The system performance decreases, if templates are stored randomly as compared with if templates are stored in a particular manner. To store the templates in a proper way, Sir Henry [2] has given a classification scheme. The following section highlights the Henry classification scheme.

\section{HENRY CLASSIFICATION SCHEME}

The Henry classification scheme allows for logical categorization of fingerprint records into Primary Group Ratio (PGR) based on fingerprint pattern types [2]. To find the PGR values, Henry classification scheme assigns each finger a number according to the order in which it is located in the hand, beginning with the right thumb as number 1 and ending with the left pinky/little as number 10 as shown in Table 1.

Table 1. Henry Classification Table

\begin{tabular}{|c|c|c|c|c|c|c|c|c|c|c|}
\hline & $\mathbf{R}$ & $\mathbf{R}$ & $\mathbf{R}$ & $\mathbf{R}$ & $\mathbf{R}$ & $\mathbf{L}$ & $\mathbf{L}$ & $\mathbf{L}$ & $\mathbf{L}$ & $\mathbf{L}$ \\
Thumb & Index & Middle & Ring & Pinky & Thumb & Index & Middle & Ring & Pinky \\
\hline $\begin{array}{c}\text { Finger } \\
\text { Number }\end{array}$ & 1 & 2 & 3 & 4 & 5 & 6 & 7 & 8 & 9 & 10 \\
\hline $\begin{array}{c}\text { Value } \\
\text { (ff Whorl) }\end{array}$ & 16 & 16 & 8 & 8 & 4 & 4 & 2 & 2 & 1 & 1 \\
\hline $\begin{array}{c}\text { Pattern } \\
\text { Type }\end{array}$ & loop & whorl & arch & arch & Loop & arch & loop & whorl & loop & arch \\
\hline $\begin{array}{c}\text { Finger } \\
\text { Value }\end{array}$ & 0 & $\mathbf{1 6}$ & 0 & 0 & 0 & 0 & 0 & $\mathbf{2}$ & 0 & 0 \\
\hline
\end{tabular}

Henry also assigns a numerical value to fingers that contain a whorl pattern; fingers 1 and 2 each have a value of 16, fingers 3 and 4 have a value of 8 , and so on, with the last two fingers having a value of 1 .

Fingers with a non-whorl pattern, such as an arch or loop pattern, have a value of zero. Both palm prints of a person (as shown in Figure 2) are required to apply the Henry classification scheme.

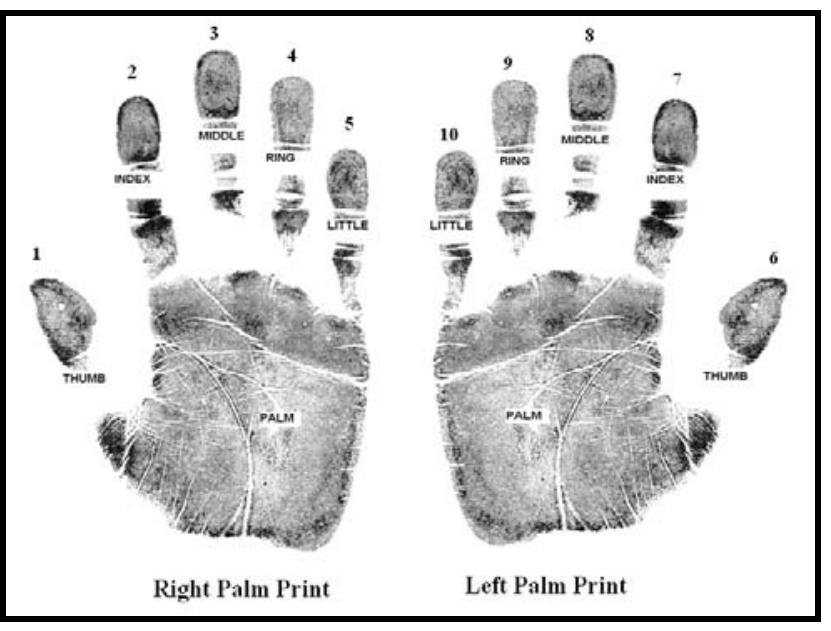

Fig. 2 - Palm prints of a single person
According to the Henry classification scheme, fingerprints are stored in the database with their relative PGR values. The fingerprint Primary Group Ratio is determined by calculating the ratio of one plus the sum of the values of the whorl-patterned, even-numbered fingers; divided by one plus the sum of the values of the whorl-patterned, odd-numbered fingers (Harling 1996). Mathematically the formula can be written as:

PGR $=\frac{1+. .(\text { Sum of EVEN }, \text { whorlder,finger values })}{1+. .(\text { Sum of ODD, whorlder,finger vale })}$

To illustrate the computation of PGR values, consider an individual having a fingerprint record with a LWAALALWLA pattern series (where L stands for Loop, W stands for Whorl and A stands for Arch). The series begins with Finger 1 (i.e. right thumb) and ending with Finger 10 (i.e. left pinky finger). By referring to table 4.1 the weights of even finger having whorl pattern are 16 and 2 (which have been shown as bold in Table 4.1). Further no odd finger has whorl pattern in the given series, hence the sum of its value is nil.

$$
\text { PGR }=\frac{1+. .(\text { Sum of EVEN }, \text { whorlder, finger values })}{1+. .(\text { Sum of ODD }, \text { whorlder, finger value })}=\frac{1+(16+2)}{1+(0)}=\frac{19}{1}
$$


Therefore, this individual belongs to the 19:1 primary group. This PGR ratio is always treated as 19/1 not 19, as PGR contains both numerator and denominator. In case an individual does not have any whorl-patterned fingerprints, his classification ratio, or primary group, would be 1:1. On the other hand if an individual having all ten fingerprints containing a whorl pattern, his classification ratio would be 31:31. Henry classification scheme reduces the effort to search a large templates of database, as fingerprints are now stored with their respective PGR values. However Henry classification scheme has some limitations as given below:

(i) Henry classification scheme work, only when both palm-prints of person are stored in database. The scheme could not work with single fingerprint template.

(ii) The problem can also arise if the criminal as made some trick while enrolling his palm-print to system. For instance, he can change the normal order of his fingers on the sensor. (ii) Amount of computer memory increases to store both palms of person as compared to single fingerprint template.

\section{THE PROPOSED METHOD TO SPEED- UP FINGERPRINT MATCHING PROCESS}

Here we present a method based on the theory of fingerprint classification to address the problem stated above. Unlike to the Henry classification, the proposed approach can work with a single fingerprint. This single print can be a thumb print or print of an index finger. In a conventional system, the database contains the fingerprint templates in an ordinary manner [3]. But in the proposed system the database contains the different set of templates according to their classification that is achieved during the enrollment process. The schematic diagram of the proposed approach is shown in Figure 3 and the enrollment/identification process of the approach is described below:

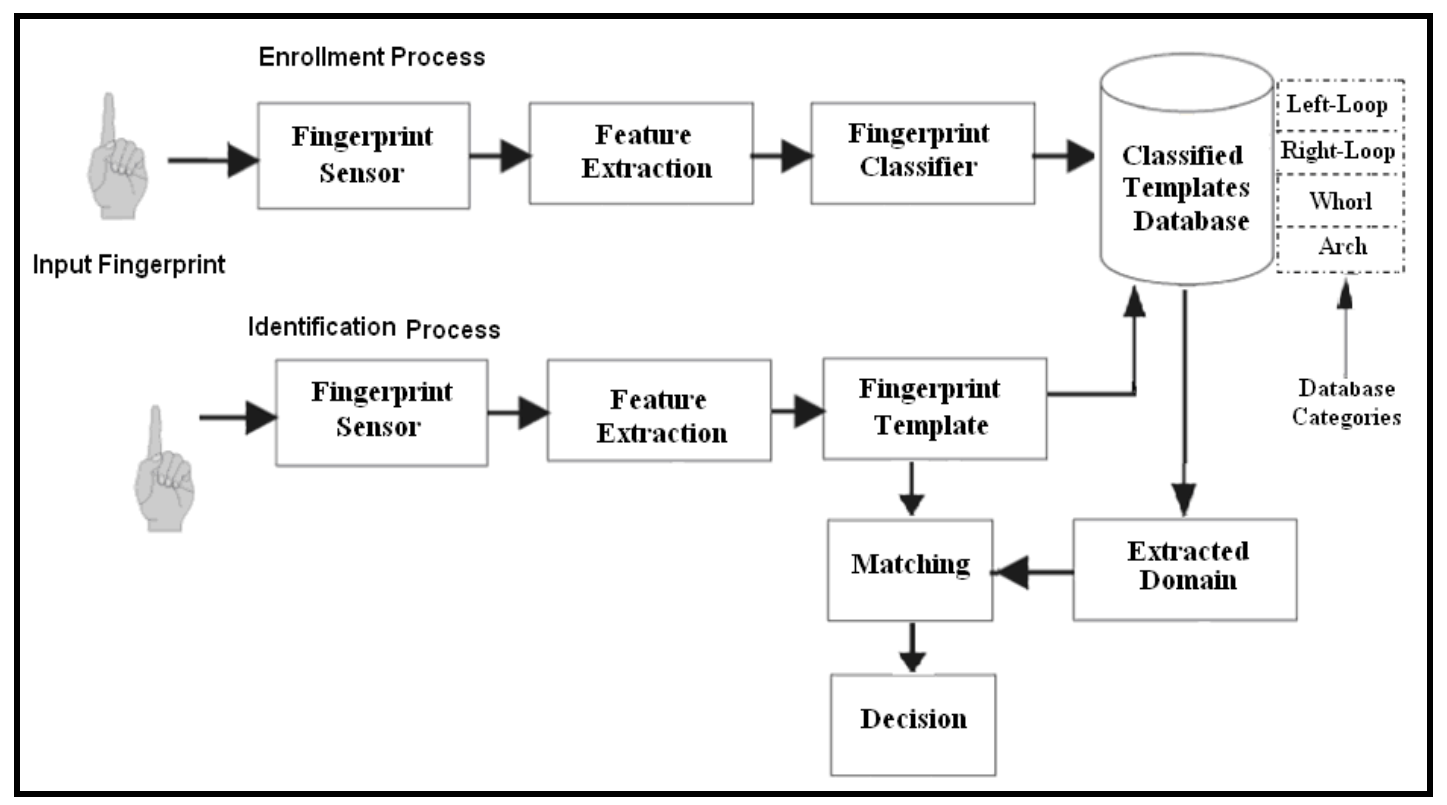

Fig. 3 - Proposed Classifier for Fingerprint Identification

\subsection{ENROLLMENT PROCESS}

(i) Sensor Module, senses the Fingerprint Templates

(ii) Feature Extraction Module, extracts the minutiae points from Fingerprint Template.

(iii) Fingerprint Classifier Module, classifies the input templates based on Left-Loop, RightLoop, Whorl or Arch and store them into the database.

\subsection{IDENTIFICATION PROCESS}

(i) Sensor Module senses the input Fingerprint Template.

(ii) Feature Extraction Module, extracts the minutiae points from Fingerprint Template.

(iii) A minutiae template has been generated of a particular classification and search the database for its specific domain. The particular domain is then extracted from database and matched with the Minutiae Template as shown in Figure 3 above. 
In the database classification, only whorl, arch and loops are considered because loops make up nearly $65 \%$ of all fingerprints, whorls are nearly $30 \%$, and perhaps $5 \%$ are arches [4]. In the proposed approach, loops are further divided into two categories viz. left-loop and right-loop as loops contribute $65 \%$ of the total fingerprints. Consequently, there become four domains i) LeftLoop ii) Right-Loop iii) Arch and iv) Whorl as shown in figure 4 below.

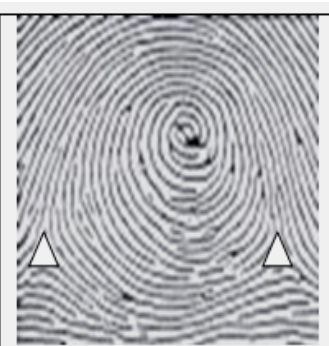

Whorl

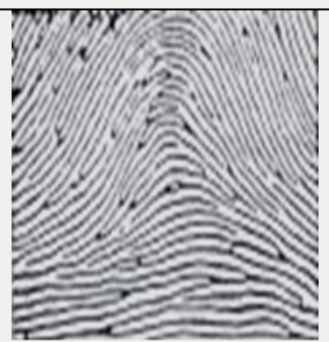

Arch

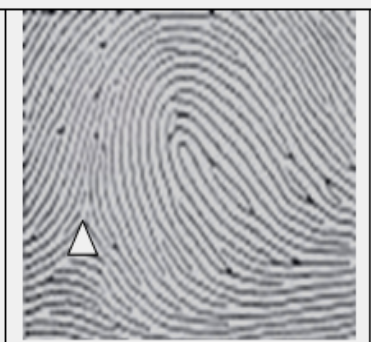

Right-Loop

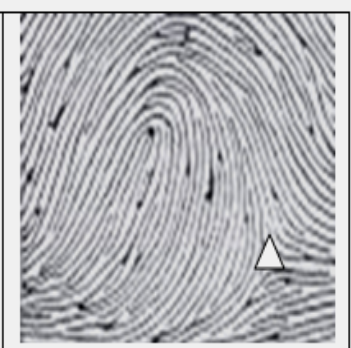

Left-Loop

Fig. 4 - Position of Deltas in different Fingerprints

The proposed classifier classifies the input templates into four categories (mentioned above) on the basis of singular points. Various singular points in any fingerprint are as given in Figure 5. Many types of minutiae's exist, including dots (very small ridges), islands (ridges slightly longer than dots, ponds or lakes (empty spaces between two temporarily divergent ridges), spurs (a notch protruding from a ridge), bridges (small ridges joining two longer adjacent ridges), and crossovers (two ridges which cross each other) [5].

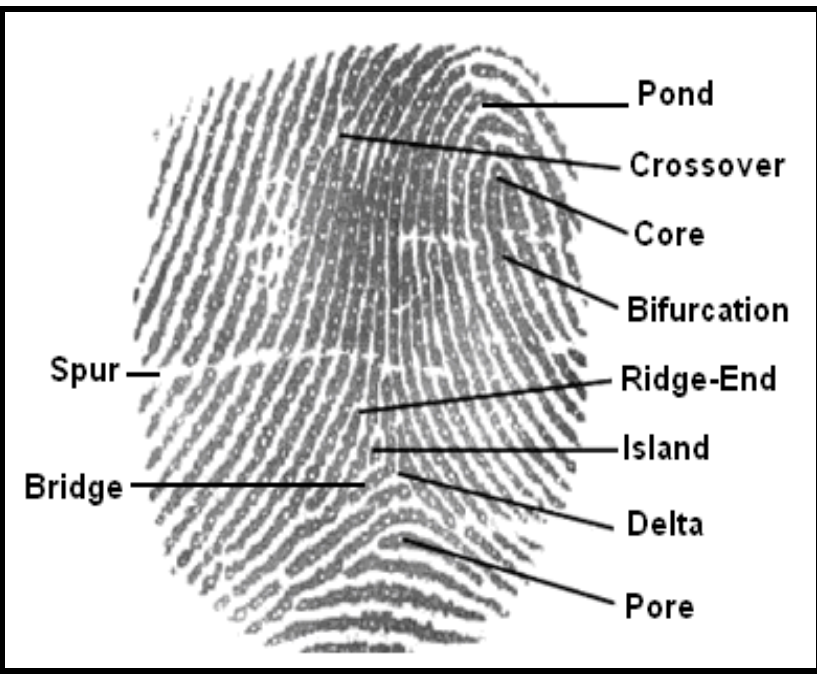

Fig. 5 - Minutiae- points on a fingerprint

The algorithm for the proposed classifiers is shown in Figure 6. If the value of $\mathrm{N}_{D}$ (i.e. number of deltas) is 2 then it is either taken as whorl or twin loop. If the value of $\mathrm{N}_{\mathrm{D}}$ is 1 then it is further computed for Left-Loop or Right-Loop. If the value of $N_{D}$ is 0 then it is marked as Arch. To find the category of loop (right or left) the feature extraction stage estimates an axis locally symmetric to the ridge structures at the core and computes $\gamma$ (number of ridges crossing the line segment joining core and delta). The relative position, $\mathrm{R}$, of delta with respect to symmetry axis is determined as follows $\mathrm{R}=1$ if the delta is on the right side of symmetry axis and it will be left loop otherwise $\mathrm{R}=0$ and in this case it will be a right loop [6]. In this way we find the categories of input fingerprint and build our database containing different domains of fingerprints. Now let's find the efficiency of proposed approach.

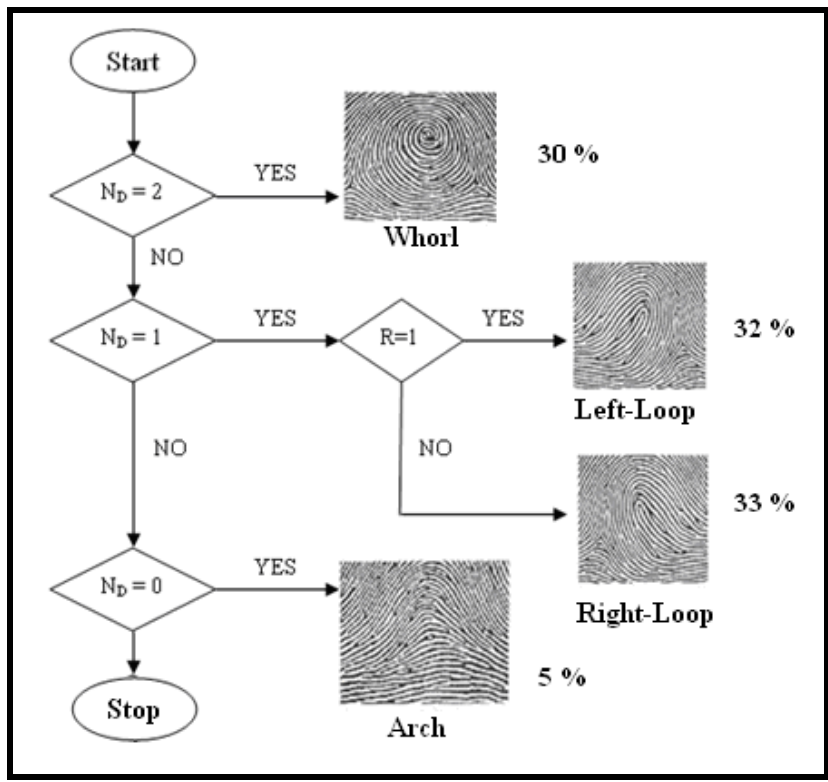

Fig. 6 - Working of proposed Fingerprint classifier

The proposed approach proves very efficient especially when the stored database is very large that is having more than 1,00000 templates.

The proposed method was evaluated at Madhuban Forensic Laboratory, Karnal, where the system database contains more than 1,50000 
templates. The software used at Madhuban Forensic Laboratory is FACTS (Finger Analysis Criminal Tracing System) developed by CMC, New Delhi based on Henry classification scheme as discussed above.

The experiment was conducted on FACTS with an input template (my own fingerprint). The system took 22.35 minutes to produce output. There were 34 templates in output. These outputs are further subjected to human verification and that took 4.55 hours to give the exact identification. Therefore it is obvious that the existing classification scheme is very time consuming and involves complication of human verification. Two templates are declared as matched if their Spatial distance and Direction are below than specified values as given by L. Hong [5]. The algorithm is discussed below:

Let $\mathrm{D}$ and $\mathrm{T}$ be the representation of the Database Template and Target Template respectively. Each minutia may be described by a number of attributes, including its location in the fingerprint image, orientation, type etc. Most common minutiae matching algorithms consider each minutiae as a triplet $\mathrm{m}=\{\mathrm{x}, \mathrm{y}, \theta\}$ that indicates the minutiae location coordinates and the minutiae angle $\theta$.

$$
\begin{gathered}
\mathrm{D}=\left\{\mathrm{m}_{1}, \mathrm{~m}_{2}, \ldots \ldots . \mathrm{m}_{\mathrm{n}}\right\} \quad \mathrm{m}_{\mathrm{i}}=\left\{\mathrm{x}_{\mathrm{i}}, \mathrm{y}_{\mathrm{i}}, \theta_{i}\right\} \quad \mathrm{i}=1 \ldots \mathrm{m} \\
\mathrm{T}=\left\{\mathrm{m}^{\prime}{ }_{1}, \mathrm{~m}^{\prime}{ }_{2}, \ldots \ldots . \mathrm{m}^{\prime}{ }_{\mathrm{n}}\right\} \quad \mathrm{m}_{\mathrm{j}}=\left\{\mathrm{x}_{\mathrm{j}}, \mathrm{y}_{\mathrm{j}}, \theta^{\prime}\right\} \quad \mathrm{j}=1 \ldots \mathrm{n}
\end{gathered}
$$

Where $\mathrm{m}$ and $\mathrm{n}$ denotes the number of minutiae in $\mathrm{D}$ and $\mathrm{T}$ respectively.

Database Template and Target Template and stored template will be matched, If we calculate Spatial Distance (SD) and direction difference (DD) that will below than specified value $\mathrm{r}_{0}$ and $\theta_{0}$ or we can write as [5].

$$
\operatorname{SD}\left(\mathrm{m}^{\prime}{ }_{1}, \mathrm{~m}_{1}\right)=\operatorname{sqrt}\left[\left(\mathrm{x}^{\prime}{ }_{\mathrm{i}}-\mathrm{x}_{\mathrm{i}}\right)^{2}+\left(\mathrm{y}_{\mathrm{i}}{ }-\mathrm{y}_{\mathrm{i}}\right)^{2}\right]<=\mathrm{r}_{0}
$$

Similarly

$$
\mathrm{DD}\left(\mathrm{m}^{\prime}, \mathrm{m}_{1}\right)<=\theta_{0}
$$

Where $\mathrm{r}_{0}$ and $\theta_{0}$ are minimum threshold values.

Now the efficiency of proposed system is computed as follows:

Assume one match takes $1 \mathrm{~ms}$ time and there are
1,50000 templates in the database.

Calculation for the best case:

The template is first match, time required $=1 \mathrm{X} 1=1 \mathrm{~ms}$.

Calculation for the worst case:

The database templates $(1,50000$ templates) are classified into the four domains (Whorl domain, Left-Loop domain, Right-Loop domain and Arch domain) as discussed in section 4.2.5 are computed below:

Number of templates in Whorl-domain $=45000$ templates.

(That is $30 \%$ of 1,50000 ).

Number of templates in Left-Loop-domain $=$ 48000 templates.

(That is $32 \%$ of 1,50000 ).

Number of templates in Right-Loop-domain: 49500 templates.

(That is $33 \%$ of 1,50000 ).

Number of templates in Arch-domain: 7500 templates.

(That is $5 \%$ of 1,50000 ).

The input template may belong to either whorl domain or left-loop domain or right-loop domain or arch domain. Now the amount of time required to match the input template with the database template is computed below:

If input template is found in Whorl domain, It takes $=1 \mathrm{~ms} X 45000=45 \mathrm{sec}$.

If input template is found in Left-Loop domain, It takes $=1 \mathrm{~ms} X 48000=48 \mathrm{sec}$.

If input template is found in Whorl domain, It takes $=1 \mathrm{~ms} \mathrm{X} 49500=49.5 \mathrm{sec}$

If input template is found in Whorl domain, It takes $=1 \mathrm{msX} 7500=7.5 \mathrm{sec}$.

Average time for worst case $=(45+48+49.5+7.5) / 4=150 / 4=37.4 \mathrm{sec}$.

\section{Efficiency of Existing System:}

Calculation for the best case:

The template is First match, Time required $=1 \mathrm{X} 1=1 \mathrm{~ms}$

Calculation for the worst case:

The template is last match, Time required $=1 X 1,50000=150 \mathrm{sec} .=25 \mathrm{~min}$.

$$
\text { Speedup Factor }=\frac{\text { Time taken by proposed approach }}{\text { Time taken by existing approach }}=\frac{37.4 \mathrm{sec} .}{25 \mathrm{~min}}=\frac{1}{40.1}
$$

This proves that the proposed approach is 40 times better than the existing Henry classification scheme. The other obvious advantages of proposed approach are (i) it considerably reduces the amount of memory required to store the fingerprint template as only one print is stored instead of 10 prints of palms for an individual (ii) The criminal can not fool the system as there is no order of fingerprints now. Still there are some problems in existing system. In proposed system database contain only single 
fingerprint instead of all the fingerprints of both palm, due to which the system may not work if we try to match with input finger as middle finger or little finger as they are not stored in database.

\section{CONCLUSION}

In this paper we have proposed architecture to improve the efficiency of the biometric systems based on Henry finger print classification. The proposed classifier, classifies the input template into the four domains on the basis of left-loop, rightloop, whorl or arch during the enrolment process in order to create the template database, which is vertically classified into these four domains. During identification process, a minutiae template is generated of a particular classification domain and that template is searched in the corresponding domain of the template database thereby reducing the response time of the biometric system. Theoretically, it has been shown that the proposed approach is approximately forty times more efficient as compared with the existing approaches.

\section{REFERENCES}

[1] A. K. Jain, R. Bolle, S. Pankanti (eds). Biometrics: Personal Identification in Networked Society. Kluwer Academic, December 1998.

[2] The Henry Classification System. Copyright (C) 2003 International Biometric Group.
[3] A. K. Hrechak and J. A. McHugh. Automated Fingerprint Recognition using Structural Matching. Pattern Recognition, (23) 8 (1990).

[4] American University of Beirut, Faculty of Engineering And Architecture, Department of Electrical and Computer Engineering, EECE 695 - Adaptive Filtering and Neural Networks Fingerprint Identification - project 2.

[5] L. Hong. Automatic Personal Identification Using Fingerprints. $\mathrm{PhD}$ Thesis, Michigan State University, 1998.

[6] Anil Jain Sharath Pankanti. Fingerprint Classification and Matching. 2004.

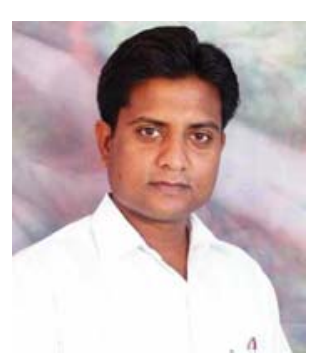

Chander Kant has Ph.D. (Computer Science) in the field of Biometrics Security; M.Tech Computer Science from Guru Jambheshwar University, Hisar; Master of Information Technology from Guru Jambheshwar University, Hisar, India.

Since 2004 he is a lecturer at Department of Computer Sciences and Applications, Kurukshetra University, India.

His research interests include: Biometric, Software Engineering, JAVA and Internet, WebEngineering, Computer Networks, Computer Graphics. 